Plant Tissue Cult. \& Biotech. 31(1): 43-49, 2021 (June)

(C)Bangladesh Assoc. for Plant Tissue Culture \& Biotechnology

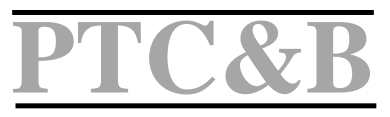

\title{
Impact of Encapsulation on Plantlet Regeneration from in vitro Grown Shoot tips of Citrus aurantifolia (Lime)
}

\author{
Priyanka Sharma* and Bidhan Roy ${ }^{1}$ \\ Department of Seed Science and Technology, Faculty of Agriculture, Uttar Banga Krishi \\ Viswavidyalaya, Pundibari, Cooch Behar, 736165, West Bengal, India
}

Key words: Citrus aurantifolia, Germination, In vitro mass propagation, Plant propagules, Plant growth regulators, Synthetic seed

\begin{abstract}
In order to conserve diverse species of citrus, an experiment on in vitro micropropagation and production of synthetic seeds from in vitro regenerated plant propagules of the species; Citrus aurantifolia (Lime) was carried out in which shoot tips were found to be suitable for excapsulation of artificial seeds. Highest rate of germination was obtained from the shoot tips when MS was supplemented with $1 \mathrm{mg} /$ BAP. Beaded shoot tips produced maximum germination $(81.43 \%)$. Germinated synthetic seeds with well established roots and shoots were were taken out from the culture bottles and transferred in plastic cups containing a mixture of sterile soil: sand and farmyard manure at a ratio of 1:1:1. Seedlings were further shifted in earthen pots and kept in a partial shed net house for 7 days. Those seedlings were finally transferred under the field conditions for acclimatization.
\end{abstract}

\section{Introduction}

The North Eastern region of India is a part of the centre of origin and is rich in diversity of citrus with wild and endangered species. The present situation of citrus genetic diversity is in an alarming rate due to its enormous destruction in the natural habitat. $C$. aurantifolia (Lime) is one of the commercially important citrus fruits, but they are grown on relatively smaller scales. Citrus is being propagated mainly by conventional techniques and is usually dependent on particular season and availability of mother plant, which restricts faster adoption and replacement of new varieties due to unavailability of sufficient quantity of propagules (Rathore et al. 2007, Goswami et al. 2013, Fanta et al. 2016).

*Author for correspondence: <sprianca133@gmail.com>

DOI: https://doi.org/10.3329/ptcb.v31i1.54110 
In vitro mass-multiplication using biotechnological tools is a powerful alternative for propagation. There are different biotechnological tools for rapid mass-multiplication of Citrus spp. viz., somatic embryogenesis, adventitious shoot bud production, and axillary enhancement which are being routinely used. Amongst these, axillary bud proliferation (micropropagation from nodal sections and shoot tips culture) is commonly practiced for in vitro mass-multiplication of Citrus because it ensures maximum genetic uniformity of the resulting plants (Rathore et al. 2007, Goswami et al. 2013). In addition, in vitro grown plantlets are apparently considered to be diseased free (Grosser and Chandler 2000).

Considering the importance of Citrus wealth of East and North-Eastern states of India, major emphasis had been laid on standardization of biotechnological tools for direct mass-multiplication through synthetic seed production using shoot tips of popular species of citrus- C. aurantifolia (Lime).

Synthetic seed is produced by enclosing viable plant materials such as somatic embryos, androgenic embryos, pre-embryos, embryos-like-structure (Roy and Mandal 2006, 2008) and protocorms (Bhattacharjee et al. 1998), protocorm-like-bodies (Balilashaki et al. 2015), axillary buds (Ganapathi et al. 1992), meristem (Kamada et al. 1989), shoot segments (Brischia et al. 2002), shoot tips (Gholami and Kavani 2018), etc. in alginate with nutrient sources. For encapsulation, plant propagules were mixed with sterilized sodium alginate $(3 \% \mathrm{w} / \mathrm{N})$ solution, particularly prepared in suitable tissue culture basal medium and supplemented with sucrose. Propagules were then picked up individually and dropped into sterilized aqueous solution of $3 \%(\mathrm{w} / \mathrm{v})$ calcium salt solution $[\mathrm{CaCl} 2$ or (CaNO3)2] with occasional agitation (Roy and Mandal 2008). Calcium alginate beads were formed within 15-30 minutes. Size of the beads depends upon the inner diameter of the pipette nozzle. In the present experiment, shoot tips were considered to be most suitable explants for encapsulation and preparation of synthetic seeds as it exhibited true-to-type planting materials.

Shoot tips which convert into plantlet directly without callus formation would reduce the risk of somaclonal variation (Wang and Den 2001) thereby making the plantlets available round the year (Reed 2002). In this endeavor, an effort was taken to standardize the protocol for preparation of synthetic seeds from shoot tips of in vitro grown plantlets of C. aurantifolia (Lime).

\section{Materials and Methods}

Seeds were collected and extracted manually from matured fruits of $C$. aurantifolia (Lime) and were cut with a sharp knife. Extracted seeds were surface sterilized with $0.1 \% \mathrm{HgCl}_{2}$ for 10 minutes followed by 3 - 5 times washing with sterilized distilled water. De-coating of surface-sterilized seeds were done under the laminar airflow cabinet and inoculated on basal MS medium. The culture bottles were incubated in a culture room at $25 \pm 2^{\circ} \mathrm{C}$ with $16 / 8 \mathrm{hrs}$ light and dark phases for germination, growth, and establishment of 
seedlings. After six weeks of inoculation, emergence of in vitro grown seedlings were observed and further used as a source of explants for preparation of synthetic seeds.

MS was prepared specifically with $30 \mathrm{~g}$ of sucrose and $8 \mathrm{~g}$ of plant tissue culturegrade agar powder. The $\mathrm{pH}$ of the medium was adjusted to 5.8. Sterilization of the prepared media was obtained by autoclaving at 121 degrees Celsius under $104 \mathrm{kPa}$ for 15 minutes. This sterilized nutrient MS medium was used for further regeneration of in vitro multiple shoots from shoot tips encapsulated synthetic seeds.

Shoot portion of in-vitro grown seedlings were cut into small pieces keeping a few nodes. Shoot tips of the seedlings were also used for regeneration of multiple plantlets. An individual culture bottle was inoculated and comprised of two explants with a total of 30 explants for each treatment. The experiment was repeated two times. Inoculated culture bottles were kept in culture room at $25 \pm 2^{\circ} \mathrm{C}$ with $16 / 8 \mathrm{~h}$ light and dark phases at optimum photosynthetic flux provided by cool fluorescent lamps for sprouting and regeneration of multiple plantlets.

Preliminary step for synthetic seed production is the preparation of Sodium alginate solution $(2.5 \% \mathrm{w} / \mathrm{N})$ of Sigma Aldrich Chemical by mixing $200 \mathrm{ml}$ of liquid MS fortified with $30 \mathrm{~g}$ of sucrose. Standard protocols developed by Roy and Mandal (2008) and Roy (2020) were used for preparation of a 3.0\% aqueous solution of calcium chloride (Sigma Aldrich Chemical) in a $500 \mathrm{ml}$ conical flask. Both the solutions were autoclaved at 121 degree Celcius under $104 \mathrm{kPa}$ for 15 minutes for sterilization. Shoot tips of well-grown plantlets were cut under the laminar airflow cabinet and used as propagules for preparation of synthetic seeds by mixing with sodium alginate solution. Individual propagule was picked up with a graduated dropper and dropped in a sterile aqueous solution of calcium chloride with occasional agitation. Calcium alginate beads were formed within 15-20 minutes. Beads were taken out by decanting off $\mathrm{CaCl}_{2}$ solution, washed with sterile double distilled water, surface dried with sterilized blotting paper and temporarily placed in clean sterilized Petri plates. Freshly prepared synthetic seeds were directly transferred and cultured on MS medium fortified with different concentrations and combinations of treatments which are mentioned below. Seedling emergence and regeneration of plantlets were recorded after two weeks of inoculation. 40 beads were employed in three replications for each treatment. The experiment was conducted entirely under a controlled environment using a laminar airflow cabinet.

Treatments used were full strength MS, $1 / 2 \mathrm{MS}, 1 / 4 \mathrm{MS}, 1.0 \mathrm{mg} / \mathrm{of} \mathrm{BAP}, 2 \mathrm{mg} / \mathrm{of} \mathrm{BAP}$, $4.0 \mathrm{mg} \Lambda$ of BAP, $1.0 \mathrm{mg} \Lambda$ of IBA $+1.0 \mathrm{mg} \Lambda$ of IAA and $1.0 \mathrm{mg} \Lambda$ of IBA $+1.0 \mathrm{mg} \Lambda$ of IAA+ $1.0 \mathrm{mg} /$ of NAA.

For interpretation of results, a completely randomized design (CRD) experiment was laid out for the laboratory experiment. Data were subjected to standard statistical methods of analysis of variance (ANOVA) using Ag Res Statistical Software, (c) 1994 Pascal Intl Software Solutions, Version 3.01, and significant differences were compared 
by LSD at $\mathrm{p}=0.05$. Analysis of data was used to interpret the results and draw conclusions.

\section{Results and Discussion}

Data on germination of synthetic seeds were taken after six weeks of inoculation (Table 1). Germination of synthetic seeds of $C$. aurantifolia (Lime)derived from shoot tips varied from $64.62 \%$ to $81.43 \%$ with a grand mean of $71.36 \%$. All the treatments showed high percentage of germination. However, maximum germination was obtained on MS medium supplemented with $1 \mathrm{mg} /$ of BAP $(81.43 \%)$ which is insignificant with $2 \mathrm{mg} \Lambda$ of BAP $(79.67 \%)$. The treatments $1 \mathrm{mg} /$ of IBA $+1 \mathrm{mg} /$ of IAA $(74.50 \%), 4 \mathrm{mg} /$ of BAP $(71.33 \%)$ and $1 \mathrm{mg} /$ of IBA $+1 \mathrm{mg} \Lambda$ of IAA $+1 \mathrm{mg} /$ of NAA $(70.33 \%)$ were also found to be insignificant among themselves.

Table 1. Germination of synthetic seeds of $C$. aurantifolia (Lime) on different plant growth regulators under in vitro conditions.

\begin{tabular}{|c|c|}
\hline Treatment & Germination $(\%)$ \\
\hline MS & $64.62 \mathrm{de}$ \\
\hline $1 / 2$ MS & $66.75 \mathrm{~cd}$ \\
\hline $1 / 4 \mathrm{MS}$ & $62.26 \mathrm{e}$ \\
\hline $\mathrm{MS}+1 \mathrm{mg} \Lambda$ of $\mathrm{BAP}$ & $81.43 \mathrm{a}$ \\
\hline $\mathrm{MS}+2 \mathrm{mg} \Lambda$ of $\mathrm{BAP}$ & $79.67 \mathrm{a}$ \\
\hline $\mathrm{MS}+4 \mathrm{mg} \Lambda$ of $\mathrm{BAP}$ & $71.33 \mathrm{~b}$ \\
\hline $\mathrm{MS}+1 \mathrm{mg} \Lambda$ of IBA $+1 \mathrm{mg} \Lambda$ of IAA & $74.50 \mathrm{~b}$ \\
\hline $\mathrm{MS}+1 \mathrm{mg} \lambda$ of IBA $+1 \mathrm{mg} \lambda$ of IAA $+1 \mathrm{mg} \lambda$ of $\mathrm{NAA}$ & $70.33 \mathrm{bc}$ \\
\hline Range & $64.62-81.43$ \\
\hline Mean & 71.36 \\
\hline
\end{tabular}

Values bearing same letter in the column are not significantly different at $\mathrm{p}=0.05$ of LSD.

Germinated synthetic seeds were allowed to grow until the roots were well developed. Well grown rooted plantlets from synthetic seeds were taken out from the culture bottles. Medium that adhered with the regenerated plantlets was removed by washing in running water. The plants were transferred to the plastic cups containing a mixture of sterile sand : soil : farmyard manure at a ratio of $1: 1: 1$ and kept in the plant tissue culture chamber for two weeks. After two weeks, the cups were kept in a room condition at ambient environment for another one week (Fig. 1). Then it was shifted under partial shed-net for two weeks in earthen-pots. Acclimatized seedlings obtained from synthetic seeds were finally planted in the field condition. Based on the results of 
germination and emergence of shoots, it clearly signifies that the synthetic seed technology could be used as a true-to-type plant propagation method in massmultiplication of Citrus spp. Such kind of sodium alginate encapsulated plant propagules could be useful in exchange of sterile materials between laboratories due to its small size and relative ease in handling these structures, or in germplasm conservation with proper preservation techniques, conservation through cryo-preservation as per the justifications and observations of the following researchers (Roy and Mandal 2008, Gholami and Alavi 2016, Gholami and Kavani 2018, Amin and Mujeeb 2019) or even in plant propagation and nurseries. Similar kind of results are also in agreement by the work of several researchers such as (Piccioni and Standardi (1995). MS medium fortified with $1.0 \mathrm{mg} \Lambda$ BAP was found to be suitable for in vitro mass-multiplication of plantlets of $C$. aurantifolia (Lime) from shoot tips segments of in vitro germinated seedlings with a high germination percentage of $81.43 \%$.
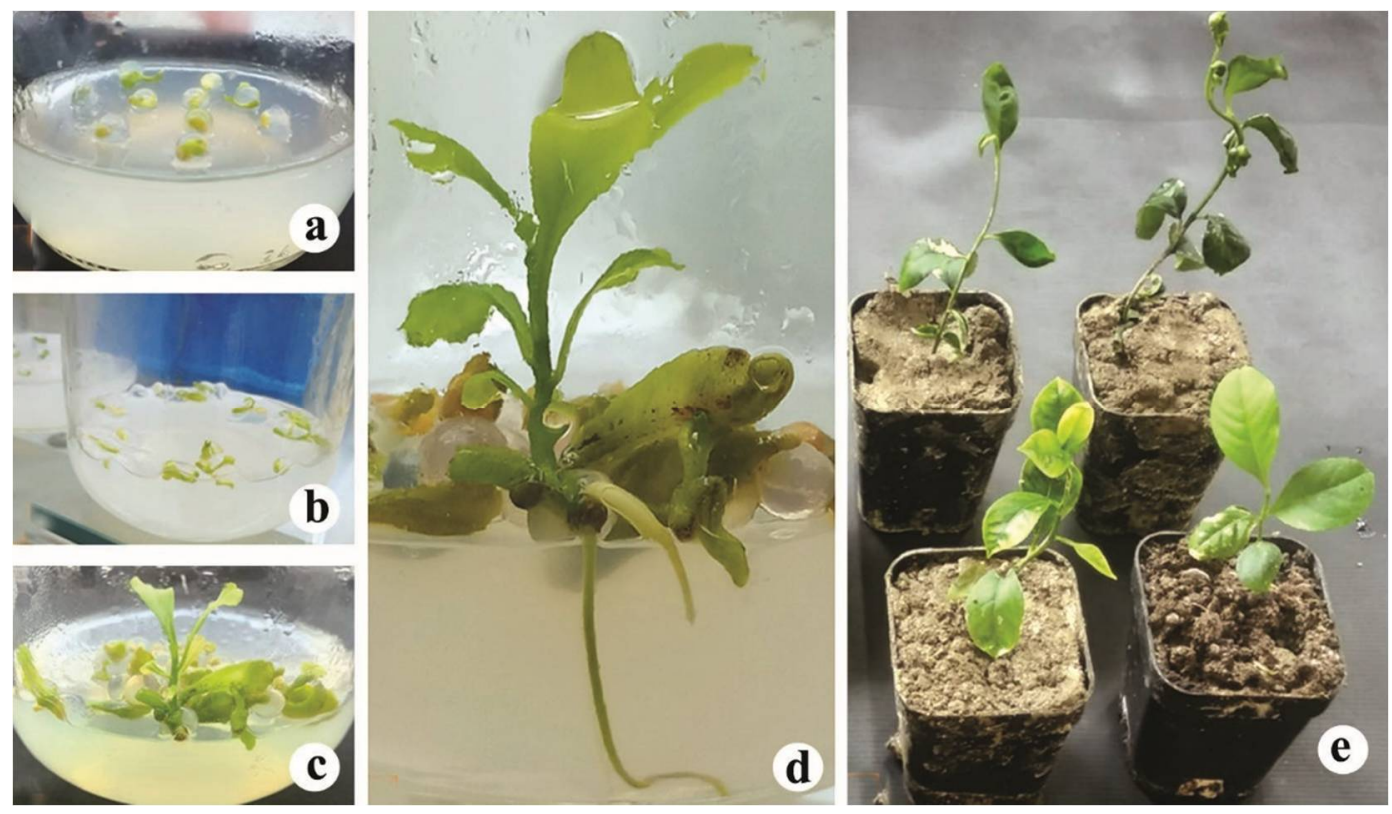

Fig. 1 (a-e). Depiction of Synthetic seeds of C. aurantifolia (Lime). (a) Inoculated synthetic seeds on MS supplemented with different combinations and concentrations of plant growth regulators (b) Germinating synthetic seeds (c) Well germinated synthetic seeds (d) Well grown plantlets obtained from synthetic seeds with roots(e) Hardening of plantlets derived from synthetic seeds.

Based on the results of the present study, it could be clearly confirmed that MS medium fortified with $1.0 \mathrm{mg} \Lambda \mathrm{BAP}$ was found to be suitable for in vitro massmultiplication of plantlets of $C$. aurantifolia (Lime) from shoot tips segments of in vitro 
germinated seedlings. Highest germination percentage of synthetic seeds was recorded when beaded shoot tips were cultured on MS medium fortified with $1.0 \mathrm{mg} / \mathrm{BAP}$ (81. $43 \%$ ). In addition, rate of germination of synthetic seeds was found to be comparatively high on all the treatments consisted of plant growth regulators and at the same time, highest amongst them was also found to be in $1 / 2$ MS supplemented media. In nutshell, synthetic seed technology could be used as a true-to-type plant propagation method in mass-multiplication of Citrus spp.

Shoot tips are suitable for encapsulation studies of artificial seeds as they possess great potential for plant development from pre-existing meristematic tissues. The results encourage the use of encapsulated unipolar explants for synthetic seed technology.

\section{Acknowledgements}

The authors highly acknowledges their gratitude to the Department of Biotechnology, Ministry of Science and Technology, Government of India for providing financial support and infrastructural facilities through sanction order No: PT/PR16132/95/ 160/2015, dated 6th January, 2017 in order to carry out the research works on diverse species of Citrus.

\section{References}

Amin M and Mujeeb A (2019) Callus Induction and Synthetic Seed Development in Draceana sanderiana Sanderex Mast: Lucky Bamboo. Biotechnol J. Int. 23(3): 1-8.

Balilashaki K, Vahedi M and Karimi R (2015) In vitro direct regeneration from node and leaf explants of Phalaenopsis cv. Surabaya. Plant Tiss Cult and Biotechnol. 25(2): 193205.

Barlass M and Skene K G M (1986) Citrus Biotechnology in Agriculture and Forestry Trees. Springer Verlag Heidelberg. Vol I. pp. 207-219.

Bhattacharjee S, Khan H A, Reddy P V and Bhattacharjee S (1998) In vitro seed germination, production of synthetic seeds and regeneration of plantlets of Phalaenopsis hybrid. Annals.Agril. Sci. Cairo. 43(2): 539-543.

Brischia R, Piccioni E and Standardi A (2002) Micropropagation and synthetic seed in M.26 application root stock (II): A new protocol for production of encapsulated diffentiating propagules. Plant Cell Tiss. and Org. Cult. 68(2): 137-141.

Fanta M, Menamo T and Bantte K (2016) Optimization of sucrose, plant hormones and photoperiod for in vitro propagation of Lemon (C. limon) and Macrophylla (C.macrophylla) using shoot tip. Adv. in Life Sci. and Technol. 47:31-39.

Ganapathi T R, Suprasanna P, Bapat V A and Rao P S (1992) Propagation of banana through encapsulated shoot tips. Plant Cell Rep. 11(11): 571-575.

Gholami A A and Alavi S V (2016) Plant regeneration of Citrus sinensis var. Thamson navel using sodium alginate-encapsulated shoot tips. Irananian J. Plant Physiol. 6(3): 1737-1743.

Gholami A A and Kavani B (2018) Somatic embryogenesis, encapsulation, cold storage and growth of hybrid Citrus [C. paradisi Macf. (Ducan) C. reticulata Blaco. (Dancy)] shoot tip segment.Indian J. Biotechnol. 17: 134-144. 
Impact of Plantlet Regeneration on Encapsulation

Goswami K, Sharma R, Singh P K and Singh G (2013) Micropropagation of seedless lemon (Citrus limon L. cv. Kaghzi Kalan) and assessment of genetic fidelity of micropropagated plants using RAPD markers. Physiol and Molecular Biol. of Plants 19(1):137-145.

Grosser J W and Chandler J L (2000) Somatic hybridisation of high yield, cold hardy and disease resistant parents for citrus rootstock improvement. Hortic Sci Biotechnol. 75: 641-644.

Kamada H, Kobayashi K, Kiyosue T and Harada H (1989) Stress induced somatic embryogenesis in carrot and its application to synthetic seed production. In vitro Cellular Dev. Biol. 25(12): 1163-1166.

Piccioni E and Standardi A (1995) Encapsulation of micropropagated buds of six woody species. Plant Cell Tiss and Org.Cult. 42(3): 221-226.

Rathore J S, Rathore M S, Singh M, Singh R P, and Shekhawat N S (2007) Micropropagation of mature tree of Citrus limon. Indian J. Biotechnol. 6(2): 239-244.

Reed B M (2002) Implementing cryopreservation for long-term germplasm preservation in vegetatively propagated species. In: Twoill LE and Bajaj YSP (ed) Biotechnology in Agriculture and Forestry 50, Cryopreservation of Plant Germplasm II.Springer Berlin Heidelberg. pp. 22-33.

Roy B and Mandal A B (2006) Rapid and recurrent mass-multiplication of androgenic embryos in indica rice. Indian J. Biotechnol. 5(2): 239-242.

Roy B and Mandal A B (2008) Development of synthetic seeds involving androgenic embryos and pro-embryos in elite indica rice. Indian J Biotechnol. 7(4): 515-519.

Roy B (2020) Artificial Seed-An Emerging Avenue of Seed Science and Applied Biotechnol.New India Publishing Agency, New Delhi. pp. 1-246.

Wang Z C and Deng X X (2001) Cryopreservation of citrus shoot-tips by vitrification and regeneration. Acta Hortic Sinica 28: 301-306.

(Manuscript received on 29/12/2020; revised on 02/04/2021) 